\title{
Silicon Chip-to-Chip Mode-Division Multiplexing
}

Baumann, Jan Markus; Porto da Silva, Edson; Ding, Yunhong; Kamchevska, Valerija; Galili, Michael; Dalgaard, Kjeld; Frandsen, Lars Hagedorn; Oxenløwe, Leif Katsuo; Morioka, Toshio

\section{Published in:}

Optical Fiber Communication Conference 2018

Link to article, DOI:

10.1364/OFC.2018.W1E.4

Publication date:

2018

Document Version

Peer reviewed version

Link back to DTU Orbit

Citation (APA):

Baumann, J. M., Porto da Silva, E., Ding, Y., Kamchevska, V., Galili, M., Dalgaard, K., Frandsen, L. H., Oxenløwe, L. K., \& Morioka, T. (2018). Silicon Chip-to-Chip Mode-Division Multiplexing. In Optical Fiber Communication Conference 2018 [Paper W1E.4] Optical Society of America. https://doi.org/10.1364/OFC.2018.W1E.4

\section{General rights}

Copyright and moral rights for the publications made accessible in the public portal are retained by the authors and/or other copyright owners and it is a condition of accessing publications that users recognise and abide by the legal requirements associated with these rights.

- Users may download and print one copy of any publication from the public portal for the purpose of private study or research.

- You may not further distribute the material or use it for any profit-making activity or commercial gain

- You may freely distribute the URL identifying the publication in the public portal 


\title{
Silicon Chip-to-Chip Mode-Division Multiplexing
}

\author{
Jan M. Baumann, Edson Porto da Silva, Yunhong Ding, Kjeld Dalgaard, Lars H. Frandsen, Leif Katsuo \\ Oxenløwe, Toshio Morioka \\ DTU Fotonik, Department of Photonics Engineering, Technical University of Denmark, Ørsteds Plads 343, DK-2800 Kgs. Lyngby, Denmark \\ jamba@fotonik.dtu.dk
}

\begin{abstract}
A chip-to-chip mode-division multiplexing connection is demonstrated using a pair of multiplexers/demultiplexers fabricated on the silicon-on-insulator platform. Successful mode multiplexing and demultiplexing is experimentally demonstrated, using the $\mathrm{LP}_{01}, \mathrm{LP}_{11 \mathrm{a}}$ and $\mathrm{LP}_{11 \mathrm{~b}}$ modes. (C) 2018 The Author(s)

OCIS codes: (060.4230) Multiplexing; (060.4510) Optical communications
\end{abstract}

\section{Introduction}

Space-division multiplexing (SDM) in optical fibers has been a research area in focus in the last years, as an additional way of satisfying the increasing demand in data transmission capacity by utilizing space as an additional dimension for multiplexing by using multicore and multimode fibers [1]. Mode-division multiplexing (MDM) using few mode fibers (FMFs) is attractive because of high spatial mode density, possibly easy fabrication of fibers and amplifiers and cylindrical symmetry of the fibers, which do not require precise rotational alignment during interconnection and splicing. However, due to the mode coupling in an FMF, multiple-input multiple-output digital signal processing (MIMO-DSP) is normally needed for detection [1], which is not desirable for some applications, such as data center networks, because it is costly, energy consuming and may introduce additional latency. Thus, for data center networks, it is attractive to take a MIMO-free approach. This can be achieved by using FMFs and MDM devices that have good crosstalk performance, in typical fiber lengths of up to a few kilometers, which are used in data centers.

Having an efficient mode (de)multiplexer, which can launch orthogonal linearly polarized $\left(\mathrm{LP}_{\mathrm{xy}}\right)$ modes into an FMF with low insertion loss and low crosstalk is an essential component for MDM. Several non-integrated technologies, e.g. using photonic lanterns [2] or multi-plane light conversion[3] have already been demonstrated, as well as integrated solutions [4]. In order to make MDM transceivers commercially attractive as well as to be able to integrate them with other devices, it is preferable to develop integrated solutions using photonic integrated circuits (PICs), preferably on the silicon-on-insulator (SOI) platform. By using photonic integration and vertical couplers, mode multiplexers and demultiplexers can be made compact, which is extremely important for building future generations transceivers. Moreover, these devices can be used as basic building blocks for fabricating more complex devices, such as switches. Coupling vertically off-chip is also useful for wafer-scale testing in higher production volumes.

In this paper, we demonstrate successful chip-to-fiber mode multiplexing and fiber-to-chip demultiplexing using the $\mathrm{LP}_{01}$ and two $\mathrm{LP}_{11}$ modes, effectively demonstrating chip-to-chip operation. We use an FMF supporting two mode groups and a pair of on-chip vertically coupling (de)multiplexers fabricated on the SOI platform. Error-free performance is obtained for the two demultiplexed spatial channels. The demonstrated performance of the on-chip mode multiplexer and demultiplexer indicates that FMF transmission utilizing integrated silicon (de)multiplexers is a feasible solution for increased capacity in data center networks.

\section{On-chip mode multiplexer and demultiplexer}

The (de)multiplexing chip [5], shown in Fig. 1a, is fabricated on a commercial SOI sample having a $250 \mathrm{~nm}$ silicon layer on top and a $3 \mu \mathrm{m}$ buried oxide layer. An aluminum back mirror is added below the PIC by using a flipbonding method to increase the efficiency of the grating couplers.5,6 The device has 8 input grating couplers through which light can be coupled into $\mathrm{LP}_{01}, \mathrm{LP}_{11}$ and $\mathrm{LP}_{21}$ modes. Both polarizations of each mode can be used as well as both LP11 orientations, making it possible to couple to 8 distinct channels (separately counting both polarization and degenerate modes). The coupling from the chip to an FMF is achieved by an array of four 2D photonic crystal grating couplers7, as shown in Fig. 1b, which create the appropriate field pattern above the array to couple into the corresponding modes of the FMF. The phase differences between the different coupling array inputs 
can be fine-tuned by heating elements in order to get the correct mode profiles.

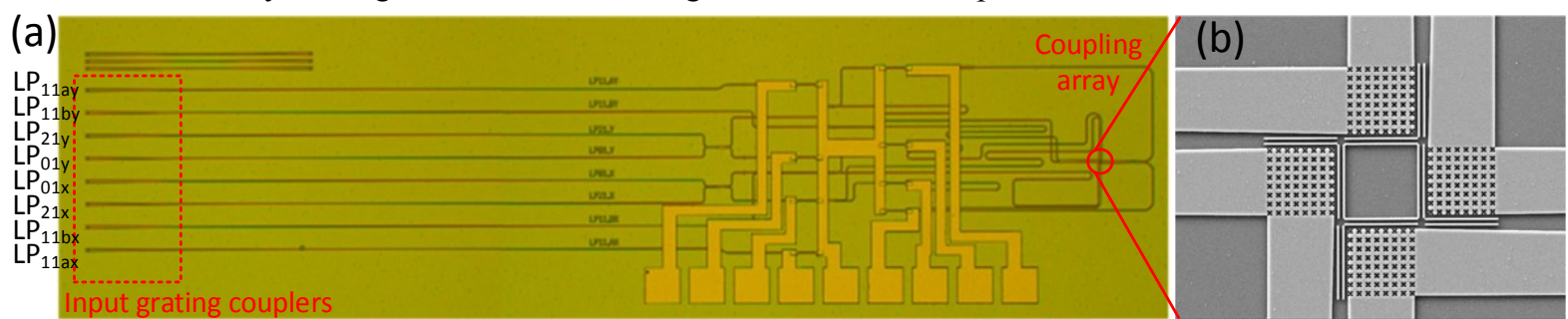

Fig. 1: (a) Image of the used multiplexing chip. (b) Image of the array of the four 2D grating couplers used to couple to an FMF. On-chip heaters are used to fine-tune phase differences between different paths.

\section{Experimental setup}

Fig. 2 shows the experimental setup, which consists of a 16 GBd QPSK transmitter and coherent receiver, and a multiplexing and identical demultiplexing chip connected using a $6 \mathrm{~m}$ long FMF. The FMF is a graded index fiber supporting only the $\mathrm{LP}_{01}$ and $\mathrm{LP}_{11}$ while having a slightly elliptical core. A $1550 \mathrm{~nm}$ laser is used to modulate and carry $16 \mathrm{GBd}$ QPSK (32 Gbit/s) data. The modulated signal is amplified by an erbium-doped fiber amplifier (EDFA) and split into four paths. Short fibers of different length $(1 \mathrm{~m}$ and $3 \mathrm{~m})$ are added in the different paths for decorrelation of the data. All signals are coupled to the grating couplers on the multiplexing chip via a fiber array. From the chip, the signals are multiplexed by the array of 2D grating couplers into different modes of the FMF, which is positioned vertically above the array. In this experiment, the signals are coupled into the $\mathrm{LP}_{01 \mathrm{y}}$, the $\mathrm{LP}_{11 a y}$ and the $\mathrm{LP}_{11 \text { ay }}$ modes, but could also be coupled into other modes of the other polarization by connecting the signals to the respective inputs of the fiber array. After the FMF, the signals are demultiplexed by a chip identical to the multiplexing chip. The demultiplexed signals are again coupled off-chip via different grating couplers into SMFs of a fiber array. A low noise amplifier is connected to one of the fiber array outputs and amplifies the signal before the receiver. At the receiver, the data is collected by an digital storage oscilloscope and processed offline.

Before characterizing the device and transmitting data, the on-chip heaters, which control the phase differences between different coupler array arms, are tuned on both the multiplexing and demultiplexing chip to create the correct field patterns above the array. The FMF is rotated above the demultiplexing chip to align the mode pattern to the chip.

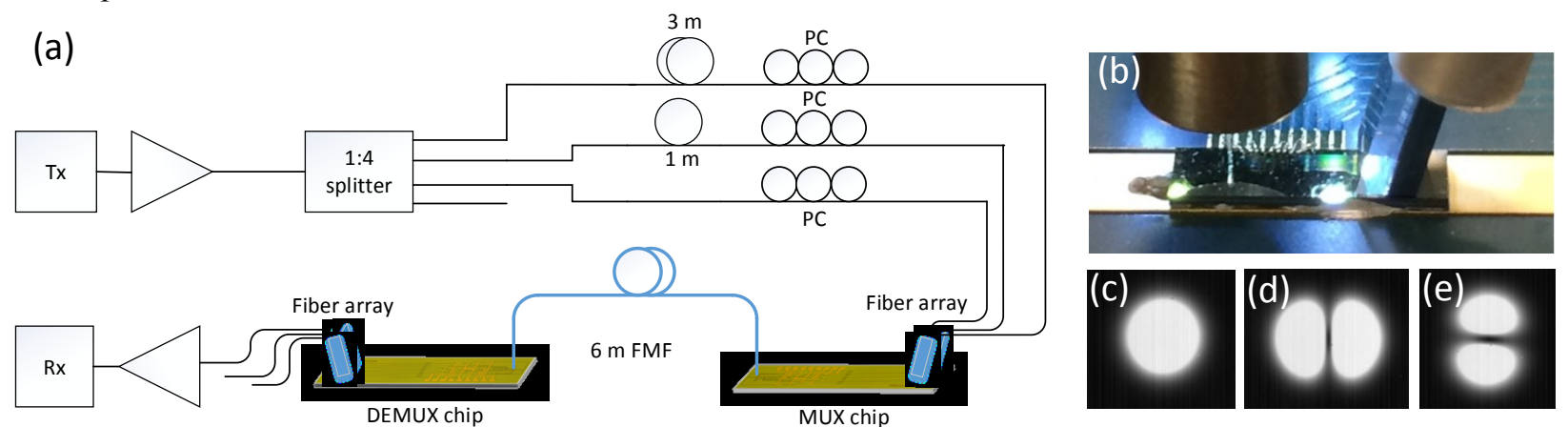

Figure 2: (a) Experimental setup: The generated signal is split up and coupled on-chip via grating couplers. The on-chip coupler array couples the signals into the different modes of a short FMF. On the demultiplexing chip, the two signals are separated again and coupled into fibers via grating couplers. The receiver unit can be connected to different chip outputs. (b): Photo of the coupling onto a multiplexing chip. (c) - (e): Mode profiles at the output of the FMF taken by an infrared camera: $\mathrm{LP}_{01 \mathrm{y}}, \mathrm{LP}_{11 \text { ay }}$ and $\mathrm{LP}_{11 \mathrm{by}}$.

\section{Results and discussion}

The transmission and crosstalk of three channels were characterized and are shown in table 1 . They are measured over the whole system, from the input fiber array of the multiplexing chip to the fiber array output after the demultiplexing chip. Light is coupled into the $\mathrm{LP}_{01 y}$, the $\mathrm{LP}_{11 \text { ay }}$ and the $\mathrm{LP}_{11 \text { by }}$ ports of the multiplexing chip and measured at the output ports of the demultiplexing chip. For the data transmission, the polarization with the highest received powers is taken as the transmission channels, due to the random orientation of the polarization to the demultiplexing chip and the lack of its control. At $1550 \mathrm{~nm}$, the $\mathrm{LP}_{01}$ channel has a transmission level of $-37.6 \mathrm{~dB}$ and crosstalks from the other inputs of below $-13.4 \mathrm{~dB}$, the $\mathrm{LP}_{11 \mathrm{a}}$ channel has a transmission level of $-33.8 \mathrm{~dB}$ and a crosstalks below $-12.1 \mathrm{~dB}$ and the $\mathrm{LP}_{11 \mathrm{~b}}$ channel has an insertion loss of $-32.9 \mathrm{~dB}$ and crosstalks below $-20.2 \mathrm{~dB}$. The different transmission of the $\mathrm{LP}_{01}$ and $\mathrm{LP}_{11}$ channels is attributed to the slightly different coupling efficiency of the (de)multiplexing chip. 
Table 1: Measured transmission and crosstalk of all channels, measured over the whole system

\begin{tabular}{|l|l|l|l|}
\cline { 2 - 4 } \multicolumn{1}{c|}{} & \multicolumn{3}{|c|}{ Output port } \\
\hline Input port & $\mathbf{L P}_{\mathbf{1 1}, \mathbf{x}}$ & $\mathbf{L P}_{\mathbf{1 1}, \mathbf{a x}}$ & $\mathbf{L P}_{\mathbf{1 1}, \mathbf{b x}}$ \\
\hline $\mathbf{L P}_{\mathbf{0 1}, \mathbf{y}}$ & $-37.6 \mathrm{~dB}$ & $<-57 \mathrm{~dB}$ & $-53.1 \mathrm{~dB}$ \\
\hline $\mathbf{L P}_{\mathbf{1 1}, \mathbf{a y}}$ & $-51.0 \mathrm{~dB}$ & $-33.8 \mathrm{~dB}$ & $-53.3 \mathrm{~dB}$ \\
\hline $\mathbf{L P}_{\mathbf{1 1} \text {, by }}$ & $-52.4 \mathrm{~dB}$ & $-45.9 \mathrm{~dB}$ & $-32.9 \mathrm{~dB}$ \\
\hline
\end{tabular}

Fig. 3 shows the measured constellation diagrams at the receiver from a 780000 bit sequence. The whole signal could be received without any errors. Out of the received data, the signal-to-noise ratio (SNR) is estimated for the different channels as $14.0 \mathrm{~dB}, 11.6 \mathrm{~dB}$ and $16.2 \mathrm{~dB}$ for the $\mathrm{LP}_{01}, \mathrm{LP}_{11 \mathrm{a}}$ and $\mathrm{LP}_{11 \mathrm{~b}}$, with measured optical signal-tonoise ratios (OSNRs) of $23.9 \mathrm{~dB}, 26.9 \mathrm{~dB}$ and $28.2 \mathrm{~dB}$. The degradation of the SNR comes mainly from the crosstalk of the other channels and limits the performance of the whole system. This can be particularly well seen in fig. 3(b), which suffers from a lot of crosstalk and where the constellation of the crosstalk can already be well seen in the constellation diagram. This penalty is mainly due to the crosstalk performance of the on-chip multiplexer and demultiplexer devices, as the used FMF is relatively short. When transmitting in a single channel without any signal in the other channels, the SNR was above $19.3 \mathrm{~dB}$ for all channels.

It was shown that mode multiplexing can be done directly chip-to-chip. Only three of the possible input channels at the multiplexer are used, because in the setup, the polarization of the arriving signal couldn't be controlled.

The received power couples to different channels and is generally spread over many outputs, if the mode patterns are not aligned with the demultiplexer (like for example if the $\mathrm{LP}_{11}$ mode rotated by $45^{\circ}$ ). An ideal demultiplexer design should be able to demultiplex from different mode orientations, otherwise the modes in the receiver need to be somehow aligned to the demultiplexer. Making the receiver invariant to the mode alignment will be subject of further research, for example using reduced MIMO processing between mode groups.

The above characterization and data transmission is mainly a characterization of the crosstalk caused by the in and out coupling of the mode multi-/demultiplexer. Transmissions over longer distances will be subject to further investigations.

A three channel, $3 \times 32 \mathrm{Gbit} / \mathrm{s}$ chip-to-chip connection has been demonstrated using the LP01 and LP11 modes in a two-mode FMF. The multiplexing and demultiplexing chips are fabricated on an SOI platform making them compatible with many other silicon photonic elements.
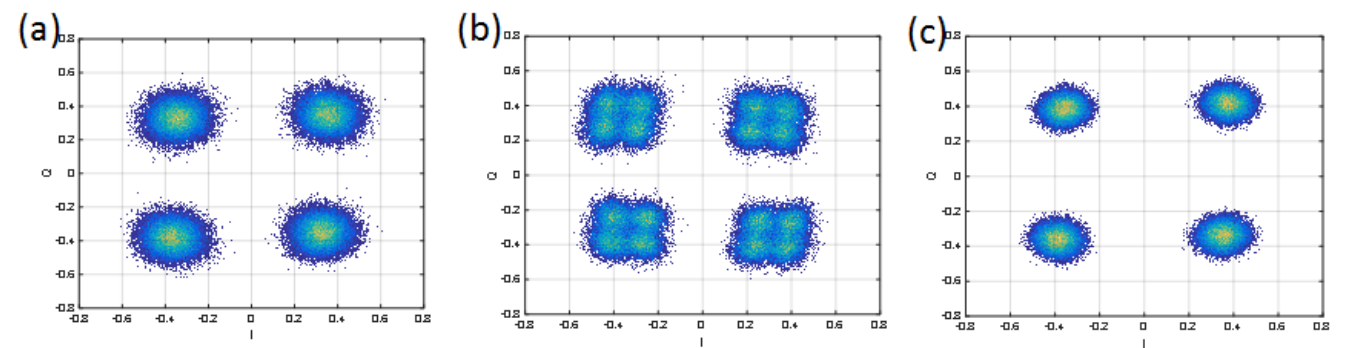

Figure 3: Signal constellation diagrams of the three different channels at the receiver after offline signal processing. The SNR was estimated out of the data: (a) LP $\mathrm{LP}_{01}$, SNR: $14.0 \mathrm{~dB}$, OSNR: $23.9 \mathrm{~dB}$, (b) LP $\mathrm{L}_{11 \mathrm{ax}}$, SNR: $11.6 \mathrm{~dB}$, OSNR: $26.9 \mathrm{~dB}$ (c) LP o1bx , SNR: $16.2 \mathrm{~dB}$, OSNR: $28.2 \mathrm{~dB}$. All three signals could be received error-free.

This work was supported by the Danish Council for Independent Research (DFF-1337-00152 and DFF-133500771) and the Center of Excellence, SPOC (Silicon Photonics for Optical Communications, ref DNRF123).

\section{References}

[1] D.J. Richardson et al., "Space-division multiplexing in optical fibres", Nature Photonics 7, 354-362 (2013)

[2] R. Ryf et al., "Mode-multiplexed transmission over conventional graded-index multimode fibers", Optics Express 23 (1), 235 (2015)

[3] G. Labroille, "Efficient and mode selective spatial mode multiplexer based on multi-plane light conversion", Opt. Express 22, 15599-15607 (2014)

[4] A.M.J. Koonen et al., "Silicon Photonic Integrated Mode Multiplexer and Demultiplexer", IEEE Photonics Technology Letters, Vol.24, No. 21, 1961-1964 (2012)

[5] Y. Ding et al., "Efficient PIC mode multiplexer using grating coupler array with aluminium mirror for few-mode fiber," CLEO 2015, paper STh1F.1. (2015). 